\title{
On the Concavity of Rate Regions for Lossless Source Coding in Networks
}

\author{
Wei-Hsin Gu \\ Department of Electrical Engineering \\ California Institute of Technology \\ Pasadena, CA 91125, USA \\ Email:whgu@z.caltech.edu
}

\author{
Michelle Effros \\ Department of Electrical Engineering \\ California Institute of Technology \\ Pasadena, CA 91125, USA \\ Email: effros@caltech.edu
}

\begin{abstract}
For a family of network source coding problems, we prove that the lossless rate region is concave in the distribution of sources. While the proof of concavity is straightforward for the few examples where a single-letter characterization of the lossless source coding region is known, it is more difficult for the vast majority of networks where the lossless source coding region remains unsolved. The class of networks that we investigate includes both solved and unsolved examples. We further conjecture that the same property applies more widely and sketch an avenue for investigating that conjecture.
\end{abstract}

\section{INTRODUCTION}

While complete, single-letter characterizations of the lossless source coding region are known for some networks, many more remain unsolved. Further, even known solutions are often characterized using auxiliary random variables; in these cases, finding the lossless rate region for a given source distribution requires solution of an often non-trivial optimization problem. Since progress in solving these problems one network at a time is slow, it is profitable to investigate properties of lossless rate regions that apply to broad classes of networks.

It is well known that the lossless rate region for a point-topoint network is concave in the source distribution. We here prove the corresponding concavity of lossless rate regions for a broad class of networks and conjecture its wider applicability.

In Section II, we formally define the network source coding problem and its lossless rate region. In Section III, we use the single-letter characterization of [1] to directly prove the concavity of the lossless rate region for the coded side information problem. In Section IV, we consider type A networks (defined in that section) and show that their lossless rate regions are also concave. In Section $\mathrm{V}$, we investigate properties of lossless rate regions for general networks that lead us to conjecture that concavity holds more widely but not for all networks.

Throughout this paper, we assume memoryless sources on finite alphabets.

\section{Problem Formulation And Notation}

We begin by defining a general lossless network source coding problem and its lossless rate region. Since we view network coding as a special case of lossless network source

This material is based upon work partially supported by NSF Grant No. CCR-0220039 and Caltech's Lee Center for Advanced Networking. coding (most authors assume independent and uniformly distributed sources when referring to network coding), we adopt many of the naming conventions from that literature.

A directed network $(\mathcal{V}, \mathcal{E})$ comprises vertex set $\mathcal{V}$ and indexed edge set $\mathcal{E} \subseteq \mathcal{V} \times \mathcal{V}$. For each edge $e=\left(v, v^{\prime}\right) \in \mathcal{E}$, we call $v$ the tail of $e$ and $v^{\prime}$ the head of $e$, denoted by tail $(e)$ and head $(e)$, respectively. The set of edges that end at vertex $v$ is denoted by $\Gamma_{I}(v)$ and the set of edges that begin at $v$ is denoted by $\Gamma_{O}(v)$, i.e.,

$$
\begin{aligned}
& \Gamma_{I}(v):=\{e \in \mathcal{E}: \operatorname{head}(e)=v\} \\
& \Gamma_{O}(v):=\{e \in \mathcal{E}: \operatorname{tail}(e)=v\}
\end{aligned}
$$

A lossless network source coding problem is defined by a 4-tuple $\mathcal{N}=(G, s, \mathcal{S}, \mathcal{T})$, where $G=(\mathcal{V}, \mathcal{E})$ is a multipath directed network, integer $s$ is the number of source random variables, and sets $\mathcal{S} \subseteq \mathcal{V} \times\{1, \ldots, s\}$ and $\mathcal{T} \subseteq \mathcal{V} \times\{1, \ldots, s\}$ describe the source availabilities and requests, respectively, so source $i \in\{1, \ldots, s\}$ is available at node $v \in \mathcal{V}$ if and only if $(v, i) \in \mathcal{S}$ and source $j \in\{1, \ldots, s\}$ is requested by node $v^{\prime} \in \mathcal{V}$ if and only if $\left(v^{\prime}, j\right) \in \mathcal{T}$. For each $v \in \mathcal{V}$, sets $I(v), J(v) \subseteq\{1,2, \ldots, s\}$ summarize $v$ 's source availabilities and requests, respectively, giving $I(v)=\{i:(v, i) \in \mathcal{S}\}$ and $J(v)=\{j:(v, j) \in \mathcal{T}\}$. We here consider only supportable network source coding problems, meaning that for any demand $\left(v^{\prime}, j\right) \in \mathcal{T}$ there exists a node $v \in \mathcal{V}$ for which $(v, j) \in \mathcal{S}$ and there exists a path from $v$ to $v^{\prime}$ in $G$.

Let $\mathbf{X}=\left(X_{1}, \ldots, X_{s}\right) \in \mathcal{X}_{1} \times \cdots \times \mathcal{X}_{s}$ be an $s$-dimensional random vector representing the sources in $\mathcal{N}=(G, s, \mathcal{S}, \mathcal{T})$, and let $P_{\mathbf{X}}$ denote its probability mass function (pmf). We also assume that $G$ is acyclic but note that cyclic graphs can be handled using the approach from [2]. Given an $|\mathcal{E}|$ dimensional rate vector $\mathbf{R}=(R(e))_{e \in \mathcal{E}}$, a rate-R, length$n$ block code $\mathcal{C}$ for $\left(\mathcal{N}, P_{\mathbf{X}}\right)$ comprises a set of encoding functions $\mathcal{F}:=\left\{f_{e} \mid e \in \mathcal{E}\right\}$ and a set of decoding functions $\mathcal{G}:=\left\{g_{v, j} \mid v \in \mathcal{V}, j \in J(v)\right\}$. The encoding and decoding functions are defined as follows

(i) For each $v \in \mathcal{V}$ with $\Gamma_{O}(v) \neq \emptyset$, every $e \in \Gamma_{O}(v)$ has 
an encoding function

$$
\begin{aligned}
f_{e}: & {\left[\prod_{e^{\prime} \in \Gamma_{I}(v)}\left\{1,2, \ldots, 2^{n R\left(e^{\prime}\right)}\right\}\right] \times\left[\prod_{i \in I(v)} \mathcal{X}_{i}^{n}\right] } \\
& \rightarrow\left\{1,2, \ldots, 2^{n R(e)}\right\} .
\end{aligned}
$$

(ii) For each $v \in \mathcal{V}$ with $J(v) \neq \emptyset$, every $j \in J(v)$ has a decoding function

$$
\begin{aligned}
g_{v, j}: & {\left[\prod_{e \in \Gamma_{I}(v)}\left\{1,2, \ldots, 2^{n R(e)}\right\}\right] \times\left[\prod_{i \in I(v)} \mathcal{X}_{i}^{n}\right] } \\
& \rightarrow \mathcal{X}_{j}^{n} .
\end{aligned}
$$

Rate vector $\mathbf{R}$ is losslessly achievable if and only if there exists a sequence of rate-R, length- $n$ block codes $\left\{\mathcal{C}_{n}\right\}_{n=1}^{\infty}$ with an asymptotically small probability of error. More precisely, for any $v \in \mathcal{V}$ with $J(v) \neq \emptyset$ and any $j \in J(v)$, if $\widehat{X}_{j}^{n}(v)$ denotes the reproduction of source $X_{j}^{n}$ at node $v$, then

$$
\lim _{n \rightarrow \infty} \operatorname{Pr}\left\{X_{j}^{n} \neq \widehat{X}_{j}^{n}(v)\right\}=0 .
$$

The closure of the set of losslessly achievable rate vectors is the lossless rate region, denoted by $\mathcal{R}_{L}\left(\mathcal{N}, P_{\mathbf{X}}\right)$. When $\mathcal{N}$ is clear from context, we shorten this notation to $\mathcal{R}_{L}\left(P_{\mathbf{X}}\right)$.

\section{AN EXAMPLE}

Consider the coded side information problem of Fig. 1.

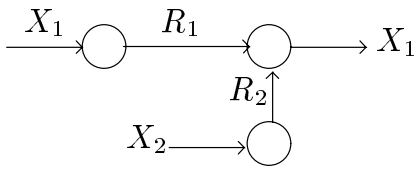

Fig. 1. Coded side-information problem.

By [1], the network's lossless rate region is

$$
\begin{aligned}
\mathcal{R}_{L}\left(P_{\mathbf{X}}\right)= & \left\{\left(R_{1}, R_{2}\right) \mid R_{1} \geq H\left(X_{1} \mid U\right), R_{2} \geq I\left(X_{2} ; U\right)\right. \\
& \text { for some } \left.\operatorname{pmf} P_{\mathbf{X}}\left(x_{1}, x_{2}\right) P_{U \mid X_{2}}\left(u \mid x_{2}\right)\right\} .
\end{aligned}
$$

Given pmfs $P_{\mathbf{X}}^{(a)}\left(x_{1}, x_{2}\right)$ and $P_{\mathbf{X}}^{(b)}\left(x_{1}, x_{2}\right)$ and any $\lambda \in$ $[0,1]$, let $P_{\mathbf{X}}=\lambda P_{\mathbf{X}}^{(a)}+\bar{\lambda} P_{\mathbf{X}}^{(b)}$, where $\bar{\lambda}=1-\lambda$. We wish to prove that $\mathcal{R}_{L}\left(P_{\mathbf{X}}\right) \subseteq \lambda \mathcal{R}_{L}\left(P_{\mathbf{X}}^{(a)}\right)+\bar{\lambda} \mathcal{R}_{L}\left(P_{\mathbf{X}}^{(b)}\right)$, where for any $A, B \subseteq \mathbb{R}^{d},(\lambda A+\bar{\lambda} B)=\{\lambda a+\bar{\lambda} b: a \in A, b \in B\}$.

For any $\mathbf{R}=\left(R_{1}, R_{2}\right)$, let $P_{U \mid X_{2}}\left(u \mid x_{2}\right)$ be a conditional pmf such that $R_{1} \geq H\left(X_{1} \mid U\right)$ and $R_{2} \geq I\left(X_{2} ; U\right)$ hold for pmf $P_{\mathbf{X}, U}\left(x_{1}, x_{2}, u\right)=P_{\mathbf{X}}\left(x_{1}, x_{2}\right) P_{U \mid X_{2}}\left(u \mid x_{2}\right)$. Define

$$
\begin{aligned}
& P_{\mathbf{X}, U}^{(a)}\left(x_{1}, x_{2}, u\right)=P_{\mathbf{X}}^{(a)}\left(x_{1}, x_{2}\right) P_{U \mid X_{2}}\left(u \mid x_{2}\right) \\
& P_{\mathbf{X}, U}^{(b)}\left(x_{1}, x_{2}, u\right)=P_{\mathbf{X}}^{(b)}\left(x_{1}, x_{2}\right) P_{U \mid X_{2}}\left(u \mid x_{2}\right) .
\end{aligned}
$$

We henceforth use superscripts $(a)$ and $(b)$ to distinguish between the marginals, entropies, and conditional pmfs corresponding to these joint pmfs on $\left(X_{1}, X_{2}, U\right)$; quantities given without superscripts use the mixture pmf $P_{\mathbf{X}, U}\left(x_{1}, x_{2}, u\right)$. Since entropy is concave and $P_{U}(u)=\lambda P_{U}^{(a)}(u)+\bar{\lambda} P_{U}^{(b)}(u)$,

$$
H(U) \geq \lambda H^{(a)}(U)+\bar{\lambda} H^{(b)}(U) .
$$

In contrast,

$$
P_{X_{2}, U}\left(x_{2}, u\right)=\left(\lambda P_{X_{2}}^{(a)}\left(x_{2}\right)+\bar{\lambda} P_{X_{2}}^{(b)}\left(x_{2}\right)\right) P_{U \mid X_{2}}\left(u \mid x_{2}\right)
$$

implies

$$
H\left(U \mid X_{2}\right)=\lambda H^{(a)}\left(U \mid X_{2}\right)+\bar{\lambda} H^{(b)}\left(U \mid X_{2}\right) .
$$

By the log-sum inequality [3, p. 29],

$$
\begin{aligned}
H\left(X_{1} \mid U\right)= & \sum_{x_{1}, u} P_{X_{1}, U}\left(x_{1}, u\right) \log \frac{P_{U}(u)}{P_{X_{1}, U}\left(x_{1}, u\right)} \\
= & \sum_{x_{1}, u}\left[\left(\lambda P_{X_{1}, U}^{(a)}\left(x_{1}, u\right)+\bar{\lambda} P_{X_{1}, U}^{(b)}\left(x_{1}, u\right)\right)\right. \\
& \left.\log \frac{\lambda P_{U}^{(a)}(u)+\bar{\lambda} P_{U}^{(b)}(u)}{\lambda P_{X_{1}, U}^{(a)}\left(x_{1}, u\right)+\bar{\lambda} P_{X_{1}, U}^{(b)}\left(x_{1}, u\right)}\right] \\
\geq & \lambda H^{(a)}\left(X_{1} \mid U\right)+\bar{\lambda} H^{(b)}\left(X_{1} \mid U\right) .
\end{aligned}
$$

Hence, defining $R_{1}^{(j)}=H^{(j)}\left(X_{1} \mid U\right)$ and $R_{2}^{(j)}=I^{(j)}\left(X_{2} ; U\right)$, $j \in\{a, b\}$, gives

$$
R_{i} \geq \lambda R_{i}^{(a)}+\bar{\lambda} R_{i}^{(b)}
$$

for $i \in\{1,2\}$. By (1), $\left(R_{1}^{(a)}, R_{2}^{(a)}\right) \in \mathcal{R}_{L}\left(P_{\mathbf{X}}^{(a)}\right)$ and $\left(R_{1}^{(b)}, R_{2}^{(b)}\right) \in \mathcal{R}_{L}\left(P_{\mathbf{X}}^{(b)}\right)$. Thus $\mathbf{R} \in \lambda \mathcal{R}_{L}\left(P_{\mathbf{X}}^{(a)}\right)+\bar{\lambda} \mathcal{R}_{L}\left(P_{\mathbf{X}}^{(b)}\right)$. This completes the proof.

\section{TyPE A NeTwORKS}

We next investigate the concavity of lossless rate regions for type A networks, which we define as follows.

Definition 1: A network $\mathcal{N}$ is said to be of type $A$ if for any two pmfs $P_{\mathbf{X}}$ and $Q_{\mathbf{X}}$ on source vector $\mathbf{X}$,

$$
\mathcal{R}_{L}\left(\mathcal{N},\left(P_{\mathbf{X}} Q_{\mathbf{X}}\right)\right)=\mathcal{R}_{L}\left(\mathcal{N}, P_{\mathbf{X}}\right)+\mathcal{R}_{L}\left(\mathcal{N}, Q_{\mathbf{X}}\right) .
$$

Thus if network $\mathcal{N}$ is of type $\mathrm{A}$, then for any source vector

$$
\mathbf{X}=\left(\left(X_{P, 1}, X_{Q, 1}\right),\left(X_{P, 2}, X_{Q, 2}\right), \ldots,\left(X_{P, s}, X_{Q, s}\right)\right),
$$

where $\mathbf{X}_{P}=\left(X_{P, 1}, \ldots, X_{P, s}\right)$ is drawn from $P_{\mathbf{X}}$ and $\mathbf{X}_{Q}=$ $\left(X_{Q, 1}, \ldots, X_{Q, s}\right)$ is drawn from $Q_{\mathbf{X}}$ and $\mathbf{X}_{P}$ and $\mathbf{X}_{Q}$ are independent, every point in the lossless rate region for $\mathbf{X}$ is the sum of points in the lossless rate regions for $\mathbf{X}_{P}$ and $\mathbf{X}_{Q}$.

While it is easy to find examples of type A networks (the point-to-point network is a trivial example with $H(\mathbf{X})=$ $\left.H\left(\mathbf{X}_{P}, \mathbf{X}_{Q}\right)=H\left(\mathbf{X}_{P}\right)+H\left(\mathbf{X}_{Q}\right)\right)$, it is important to note that there exist networks that are not of type A. Precisely,

$$
\mathcal{R}_{L}\left(\mathcal{N},\left(P_{\mathbf{X}} Q_{\mathbf{X}}\right)\right) \supseteq \mathcal{R}_{L}\left(\mathcal{N}, P_{\mathbf{X}}\right)+\mathcal{R}_{L}\left(\mathcal{N}, Q_{\mathbf{X}}\right)
$$

for any $\mathcal{N}$ since any rate- $\mathbf{R}_{P}$ coding scheme $\mathcal{C}_{P}$ for pmf $P \mathbf{X}$ and any rate- $\mathbf{R}_{Q}$ coding scheme $\mathcal{C}_{Q}$ for pmf $Q_{\mathbf{X}}$ can be concatenated to give a rate- $\left(\mathbf{R}_{P}+\mathbf{R}_{Q}\right)$ product code for $\operatorname{pmf} P_{\mathbf{X}} Q_{\mathbf{X}}$. Importantly, the reverse inclusion is not satisfied for all networks since the product code solution is not optimal in general even though $X_{P}$ and $X_{Q}$ are independent. The example in Fig. 2, a simplification of a network coding example from [4], illustrates this point. Let $P_{\mathbf{X}}$ be uniformly distributed on $(0,0)$ and $(1,0)$, giving $H_{P}\left(X_{1}\right)=1$ and $H_{P}\left(X_{2}\right)=0$. Let $Q_{\mathbf{X}}$ be uniformly distributed on $(0,0)$ and $(0,1)$, giving $H_{P}\left(X_{1}\right)=0$ and $H_{P}\left(X_{2}\right)=1$. Applying binary 
addition at node a gives a lossless code for $P_{\mathbf{X}} Q_{\mathbf{X}}$ with rate vector $\mathbf{R}=(1,1,1)$, while $\mathbf{R} \in\left(\mathcal{R}_{L}\left(\mathcal{N}, P_{\mathbf{X}}\right)+\mathcal{R}_{L}\left(\mathcal{N}, Q_{\mathbf{X}}\right)\right)$ implies $R_{(a, b)} \geq 2$. In fact, the cut-set bound is tight for this example, and the lossless rate region for a general source distribution consists of all $\left(R_{a, b}, R_{b, c}, R_{b, d}\right)$ satisfying

$$
\begin{aligned}
& R_{a, b} \geq \max \left\{H\left(X_{1}, \mid X_{2}\right), H\left(X_{2} \mid X_{1}\right)\right\} \\
& R_{b, c} \geq H\left(X_{1} \mid X_{2}\right), R_{b, d} \geq H\left(X_{2} \mid X_{1}\right) .
\end{aligned}
$$

This is one example of a network for which the lossless rate region is not concave in general. The region can, however, be written as the intersection of the two concave lossless rate regions $R_{L}^{(1)}$ and $R_{L}^{(2)}$ defined as

$$
\begin{aligned}
& R_{L}^{(1)}=\left\{\left(R_{a, b}, R_{b, c}, R_{b, d}\right) \mid R_{a, b}, R_{b, c} \geq H\left(X_{1} \mid X_{2}\right)\right\} \\
& R_{L}^{(2)}=\left\{\left(R_{a, b}, R_{b, c}, R_{b, d}\right) \mid R_{a, b}, R_{b, d} \geq H\left(X_{2} \mid X_{1}\right)\right\} .
\end{aligned}
$$

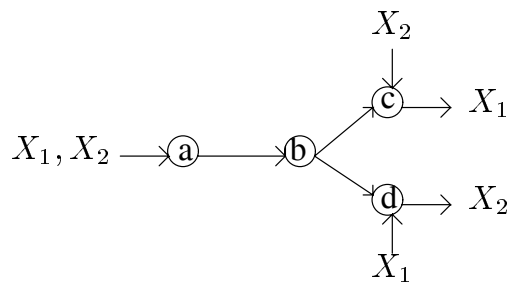

Fig. 2. An example of a network that is not concave.

We next show that the lossless rate region for any type A network is concave in the source pmf, i.e., for any two source distributions $P_{\mathbf{X}}^{(a)}$ and $P_{\mathbf{X}}^{(b)}$, and any real number $\lambda \in[0,1]$,

$$
\mathcal{R}_{L}\left(\lambda P_{\mathbf{X}}^{(a)}+\bar{\lambda} P_{\mathbf{X}}^{(b)}\right) \subseteq \lambda \mathcal{R}_{L}\left(P_{\mathbf{X}}^{(a)}\right)+\bar{\lambda} \mathcal{R}_{L}\left(P_{\mathbf{X}}^{(b)}\right) .
$$

The following characterization of $\mathcal{R}_{L}\left(P_{\mathbf{X}}\right)$ is useful for that derivation. For each $v \in \mathcal{V}$ with $J(v) \neq \emptyset$ and every $j \in J(v)$, choose one vertex $v_{v, j}^{\prime} \in \mathcal{V}$ such that $X_{j}$ can be observed by $v_{v, j}^{\prime}$ and there is a path from $v_{v, j}^{\prime}$ to $v$. Let $\mathcal{P}_{v, j}$ denote this path. For every $e \in \mathcal{E}$, let $\omega_{e}:=\left\{(v, j) \mid e \in \mathcal{P}_{v, j}\right\}$. Define

$R_{L}^{*}\left(P_{\mathbf{X}}\right)=\left\{\left(R_{e}\right)_{e \in \mathcal{E}} \mid \exists n \geq 1\right.$ and a blocklength- $n$ code for which $F_{e}$ is the output of encoder $f_{e}$ and $G_{v, j}$ is the output of decoder $g_{v, j}$ and

$$
\left.R_{e} \geq \frac{1}{n}\left(H\left(F_{e}\right)+\sum_{(v, j) \in \omega_{e}} H\left(X_{j}^{n} \mid G_{v, j}\right)\right)\right\} .
$$

Lemma 1 shows that the closure $\overline{\mathcal{R}}_{L}^{*}\left(P_{\mathbf{X}}\right)$ of $R_{L}^{*}\left(P_{\mathbf{X}}\right)$ completely characterizes the lossless rate region. While it is not a single-letter characterization, it lends useful insight into the question of concavity for type A networks.

Lemma 1:

$$
\mathcal{R}_{L}\left(P_{\mathbf{X}}\right)=\overline{R_{L}^{*}}\left(P_{\mathbf{X}}\right) .
$$

Proof. First we show that $\mathcal{R}_{L}\left(P_{\mathbf{X}}\right) \subseteq \overline{R_{L}^{*}}\left(P_{\mathbf{X}}\right)$. Given any $\mathbf{R} \in \mathcal{R}_{L}\left(P_{\mathbf{X}}\right)$ and any $\epsilon>0$, there exists a blocklength- $n$, rate-R code with encoder outputs $F_{e}$ and decoder outputs
$G_{v, j}$ such that Fano's inequality implies that for any pair $(v, j)$ such that $j \in J(v)$,

$$
H\left(X_{j}^{n} \mid G_{v, j}\right)<n \epsilon / k
$$

where $k=\sum_{v \in \mathcal{V}}|J(v)|$. Thus

$$
R_{e}+\epsilon \geq \frac{1}{n}\left(H\left(F_{e}\right)+\sum_{(v, j) \in \omega_{e}} H\left(X_{j}^{n} \mid G_{v, j}\right)\right) .
$$

Hence $\mathbf{R}+\epsilon \mathbf{1} \in R_{L}^{*}\left(P_{\mathbf{X}}\right)$, where $\mathbf{1}$ is the vector whose components are all 1 . Since $\epsilon>0$ is arbitrary, $\mathbf{R} \in \overline{R_{L}^{*}}(\mathbf{X})$. Conversely, let $\mathbf{R}=\left(R_{e}\right)_{e \in \mathcal{E}} \in R_{L}^{*}\left(P_{\mathbf{X}}\right)$ and $\epsilon>0$ be arbitrary. We aim to show that $\mathbf{R}+\epsilon \mathbf{1} \in \mathcal{R}_{L}\left(P_{\mathbf{X}}\right)$. Let $\mathcal{C}$ be a length- $n$ block code with encoding and decoding functions $\left\{f_{e}\right\}_{e \in \mathcal{E}}$ and $\left\{g_{v, j}\right\}_{v \in \mathcal{V}, j \in J(v)}$ that characterizes $\mathbf{R}$. That is, for every $e \in \mathcal{E}$,

$$
n R_{e} \geq H\left(F_{e}\right)+\sum_{(v, j) \in w_{e}} H\left(X_{j}^{n} \mid G_{v, j}\right) .
$$

For any $l$ sufficiently large, we can design a blocklength- $l$ source code to describe $l$ instances of $F_{e}$ at rate $\left(H\left(F_{e}\right)+\epsilon /(2|\mathcal{E}|)\right)$ and with error probability at most $\epsilon /(2|\mathcal{E}|)$. If $\phi_{e}^{l}$ and $\psi_{e}^{l}$ are the encoder and decoder for the source code for $F_{e}$, then

$$
\sum_{e \in \mathcal{E}} \operatorname{Pr}\left(F_{e}^{l} \neq \psi_{e}^{l}\left(\phi_{e}^{l}\left(F_{e}^{l}\right)\right)<\epsilon / 2 .\right.
$$

Similarly, when $l$ is sufficiently large, we can describe $X_{j}^{n l}$, to a decoder that knows $G_{v, j}^{l}$ at rate $H\left(X_{v, j}^{n} \mid G_{v, j}^{l}\right)+\epsilon / k$ with error probability at most $\epsilon /(2 k|\mathcal{E}|)$. If $\tau_{v, j}^{l}$ and $\mu_{v, j}^{l}$ are the encoders and decoders for this code, then

$$
\sum_{e \in \mathcal{E}} \sum_{(v, j) \in w_{e}} \operatorname{Pr}\left(X_{j}^{n l} \neq \mu_{v, j}^{l}\left(\tau_{v, j}^{l}\left(X_{j}^{n l}\right), G_{v, j}^{l}\right)\right)<\epsilon / 2
$$

for each $e \in \mathcal{E}$. Now for $e \in \mathcal{E}$, we set $\widehat{F}_{e}^{l}=\psi_{e}^{l}\left(\phi_{e}^{l}\left(f_{e}^{l}\left(\left(\widehat{F}_{e^{\prime}}^{l}\right)_{e^{\prime} \in \Gamma_{I}(\operatorname{tail}(e))},\left(X_{j}^{n}\right)_{j \in I(\operatorname{tail}(e))}\right)\right)\right)$. For any $v \in \mathcal{V}$ with $J(v) \neq \emptyset$ and $j \in J(v)$, set

$$
\widehat{G}_{v, j}^{l}:=g_{v, j}^{l}\left(\left(\widehat{F}_{e}\right)_{e \in \Gamma_{I}(v)},\left(X_{i}^{n l}\right)_{i \in I(v)}\right)
$$

and define the reproduction of $X_{j}^{n l}$ at $v$ as

$$
\widehat{X}_{j}^{n l}(v):=\mu_{v, j}^{l}\left(\tau_{v, j}^{l}\left(X_{j}^{n l}\right), \widehat{G}_{v, j}^{l}\right) .
$$

We construct a lossless source code at rate $\mathbf{R}+\epsilon \mathbf{1}$ by applying code $\left(\phi_{e}^{l}, \psi_{e}^{l}\right)$ to losslessly describe $F_{e}^{l}$ across edge $e$ and Slepian-Wolf code $\left(\tau_{v, j}^{l}, \mu_{v, j}^{l}\right)$ to losslessly describe $X_{j}$ across the edges of $w_{e}$. The event $E_{j}$ designating an error in at least one reconstruction of $X_{j}^{n l}$ has probability bounded by

$$
\begin{aligned}
\operatorname{Pr}\left(E_{j}\right) \leq & \sum_{e \in \mathcal{E}}\left[\operatorname{Pr}\left(F_{e}^{l} \neq \psi_{e}^{l}\left(\phi_{e}^{l}\left(F_{e}^{l}\right)\right)\right)+\right. \\
& \left.\sum_{(v, j) \in w_{e}} \operatorname{Pr}\left(X_{j}^{n l} \neq \mu_{v, j}^{l}\left(\tau_{v, j}^{l}\left(X_{j}^{n l}\right), G_{v, j}^{l}\right)\right)\right] \\
< & \epsilon .
\end{aligned}
$$


Letting $\epsilon \rightarrow 0$ gives $\mathbf{R} \in \mathcal{R}_{L}\left(P_{\mathbf{X}}\right)$.

Theorem 1: For a type A network,

$$
\mathcal{R}_{L}\left(\lambda P_{\mathbf{X}}^{(a)}+\bar{\lambda} P_{\mathbf{X}}^{(b)}\right) \subseteq \lambda \mathcal{R}_{L}\left(P_{\mathbf{X}}^{(a)}\right)+\bar{\lambda} \mathcal{R}_{L}\left(P_{\mathbf{X}}^{(b)}\right)
$$

for any source pmfs $P_{\mathbf{X}}^{(a)}, P_{\mathbf{X}}^{(b)}$, and $\lambda \in[0,1]$.

Proof. Let

$$
P_{\mathbf{X}}=\lambda P_{\mathbf{X}}^{(a)}+\bar{\lambda} P_{\mathbf{X}}^{(b)} \text { and } \mathbf{R}=\left(R_{e}\right)_{e \in \mathcal{E}} \in \mathcal{R}_{L}\left(P_{\mathbf{X}}\right)
$$

be given. By Lemma 1 , we pick a length- $n$ block code $\mathcal{C}$ such that

$$
R_{e} \geq \frac{1}{n}\left(H\left(F_{e}\right)+\sum_{(v, j) \in \omega_{e}} H\left(X_{j}^{n} \mid G_{v, j}\right)\right)+\epsilon \mathbf{1},
$$

where $F_{e}$ and $G_{v, j}$ are the outputs of the encoder $f_{e}$ and decoder $g_{v, j}$, respectively, of code $\mathcal{C}=\left\{\left\{f_{e}\right\},\left\{g_{v, j}\right\}\right\}$. Since $F_{e}$ is a deterministic function of $\left(\left(F_{e^{\prime}}\right)_{e^{\prime} \in \Gamma_{I}(\operatorname{tail}(e))}\right.$, $\left.\left(X_{i}^{n}\right)_{i \in I(\operatorname{tail}(e))}\right), F_{e}$ is conditionally independent of everything else given $\left(\left(F_{e^{\prime}}\right)_{e^{\prime} \in \Gamma_{I}(\operatorname{tail}(e))},\left(X_{i}^{n}\right)_{i \in I(\operatorname{tail}(e))}\right)$. Similarly, $G_{v, j}$ is conditionally independent of everything else given $\left(\left(F_{e}\right)_{e \in \Gamma_{I}(v)},\left(X_{i}^{n}\right)_{i \in I(v)}\right)$. Therefore, the conditional distribution of all encoding and decoding

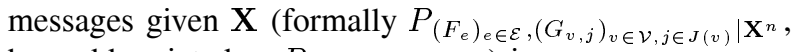
here abbreviated as $P_{\left.\left(F_{e}\right),\left(G_{v}, j\right) \mid \mathbf{X}^{n}\right)}$ is

$$
\begin{aligned}
& P_{\left(F_{e}\right),\left(G_{v, j}\right) \mid \mathbf{X}^{n}}=\prod_{e} P_{F_{e} \mid\left(\left(F_{e^{\prime}}\right)_{e^{\prime} \in \Gamma_{I}(\operatorname{tail}(e))},\left(X_{i}^{n}\right)_{i \in I(\operatorname{tail}(e))}\right)} \times \\
& \quad \prod_{(v, j): v \in \mathcal{V}, j \in J(v)} P_{G_{v, j} \mid\left(\left(F_{e}\right)_{e \in \Gamma_{I}(v)},\left(X_{i}^{n}\right)_{i \in I(v)}\right),}
\end{aligned}
$$

and the joint pmf is

$$
\begin{aligned}
P_{\mathbf{X}^{n},(} & \left.F_{e}\right),\left(G_{v, j}\right) \\
= & P_{\left(F_{e}\right),\left(G_{v, j}\right) \mid \mathbf{X}^{n}} \prod_{i=1}^{n}\left(\lambda P_{\mathbf{X}_{i}}^{(a)}+\bar{\lambda} P_{\mathbf{X}_{i}}^{(b)}\right) \\
= & P_{\left(F_{e}\right),\left(G_{v, j}\right) \mid \mathbf{X}^{n}}\left[\lambda^{n} P_{\mathbf{X}^{n}}^{(a)}+\lambda^{n-1} \bar{\lambda}\left(P_{\mathbf{X}^{n-1}}^{(a)} P_{\mathbf{X}_{n}}^{(b)}\right.\right. \\
& \left.+P_{\mathbf{X}^{n-2}}^{(a)} P_{\mathbf{X}_{n-1}}^{(b)} P_{\mathbf{X}_{n}}^{(a)}+\cdots+P_{\mathbf{X}_{1}}^{(b)} P_{\mathbf{X}_{2}^{n}}^{(a)}\right) \\
& \left.+\cdots+\bar{\lambda}^{n} P_{\mathbf{X}^{n}}^{(b)}\right] .
\end{aligned}
$$

Enumerating the pmfs on $\mathbf{X}^{n}$ as $P_{\mathbf{X}^{n}}^{(1)}, P_{\mathbf{X}^{n}}^{(2)}, \ldots, P_{\mathbf{X}^{n}}^{\left(2^{n}\right)}$ gives

$$
P_{\mathbf{X}^{n},\left(F_{e}\right),\left(G_{v, j}\right)}=P_{\left(F_{e}\right),\left(G_{v, j}\right) \mid \mathbf{X}^{n}} \sum_{i=1}^{2^{n}} a_{i} P_{\mathbf{X}}^{(i)},
$$

where $a_{1}=\lambda^{n}, a_{2}=\lambda^{n-1} \bar{\lambda}, \ldots, a_{2^{n}}=\bar{\lambda}^{n}$ are the corresponding coefficients.

For each $i \in\left\{1,2, \ldots, 2^{n}\right\}$, let $F_{e}^{(i)}$ and $G_{v, j}^{(i)}$ be random variables drawn from pmf

$$
P_{\left(F_{e}^{(i)}\right),\left(G^{(i)}\right)}=\sum_{\mathbf{x}^{n}} P_{\left(F_{e}\right),\left(G_{v, j}\right) \mid \mathbf{X}^{n}} P_{\mathbf{X}_{n}}^{(i)}\left(\mathbf{x}^{n}\right) .
$$

While we represent edge messages by random variables, recall that each $F_{e}$ is, by definition, a deterministic function of the messages and sources available to the node at its tail; thus for every $i,\left\{F_{e}^{(i)}, G_{v, j}^{(i)}\right\}$ describes a length- $n$ block code for the network. By the concavity of entropy

$$
H\left(F_{e}\right) \geq \sum_{i=1}^{2^{n}} a_{i} H\left(F_{e}^{(i)}\right)
$$

In addition, for any $v \in \mathcal{V}$ and $j \in J(v)$,

$$
\begin{aligned}
H\left(X_{j}^{n} \mid G_{v, j}\right) & \\
= & \sum_{x_{j}^{n}, g_{v, j}} P_{X_{j}^{n}, G_{v, j}}\left(x_{j}^{n}, g_{v, j}\right) \log \frac{P_{G_{v, j}}\left(g_{v, j}\right)}{P_{X_{j}^{n}, G_{v, j}}\left(x_{j}^{n}, g_{v, j}\right)} \\
= & \sum_{x_{j}^{n}, g_{v, j}}\left[\left(\sum_{i} a_{i} P_{X_{j}^{n}, G_{v, j}^{(i)}}\left(x_{j}^{n}, g_{v, j}\right)\right)\right. \\
\geq & \sum_{x_{j}^{n}, g_{v, j}} \sum_{i}\left[a_{i} P_{X_{j}^{n}, G_{v, j}^{(i)}}\left(x_{j}^{n}, g_{v, j}\right)\right. \\
& \left.\log \frac{P_{G_{v, j}^{(i)}}^{(i)}\left(g_{v, j}\right)}{\sum_{X_{j}^{n}, G_{v, j}} a_{i} P_{X_{j}^{n}, G_{v, j}^{(i)}}\left(x_{j}^{n}, g_{v, j}\right)}\right] \\
= & \sum_{i} a_{i} H\left(G_{v, j}^{(i)} \mid X_{j}^{n}\right) .
\end{aligned}
$$

by the log-sum inequality ,

Therefore, by Lemma 1 and the type A assumption,

$$
\begin{aligned}
\mathbf{R} & \in \frac{1}{n} \sum_{i} a_{i} \mathcal{R}_{L}\left(P_{\mathbf{X}^{n}}^{(i)}\right) \\
& =\frac{1}{n} \sum_{l=0}^{n}\left(\begin{array}{c}
n \\
l
\end{array}\right) \lambda^{l} \bar{\lambda}^{n-l}\left[(n-l) \mathcal{R}_{L}\left(P_{\mathbf{X}}^{(a)}\right)+l \mathcal{R}_{L}\left(P_{\mathbf{X}}^{(b)}\right)\right] \\
& =\lambda \mathcal{R}_{L}\left(P_{\mathbf{X}}^{(a)}\right)+\bar{\lambda} \mathcal{R}_{L}\left(P_{\mathbf{X}}^{(b)}\right)
\end{aligned}
$$

We end this section by showing an example of a type A network for which a single-letter characterization of the lossless rate region is currently unknown.

Consider the network shown in Fig. 3. One can easily show that there is a characterization of the lossless rate distortion, which is

$$
\begin{aligned}
& \mathcal{R}_{L}\left(P_{\mathbf{X}}\right)=\left\{\left(R_{0}, R_{1}, R_{2}\right) \mid \exists n \text { s.t. } n R_{0} \geq I\left(Z^{n} ; V\right),\right. \\
& n R_{1} \geq I\left(X^{n}, Y^{n} ; U\right)+H\left(X^{n} \mid U\right)+H\left(Y^{n} \mid U, V\right), \\
& n R_{2} \geq I\left(X^{n}, Y^{n} ; U\right)+H\left(Y^{n} \mid U, V\right) \\
& \text { where } \left.V \rightarrow Z^{n} \rightarrow\left(X^{n}, Y^{n}\right) \rightarrow U \text { is a Markov chain }\right\} .
\end{aligned}
$$

Let $\left(X^{n}, Y^{n}, Z^{n}\right)$ and $\left(\widetilde{X}^{n}, \tilde{Y}^{n}, \widetilde{Z}^{n}\right)$ be drawn i.i.d. according to $P_{\mathbf{X}}$ and $Q_{\mathbf{X}}$ respectively. For simplicity, let $T^{n}=\left(X^{n}, Y^{n}\right)$ and $\widetilde{T}^{n}=\left(\widetilde{X}^{n}, \widetilde{Y}^{n}\right)$. Now for any $n$ and any given auxiliary random pair $(U, V)$ that characterizes some rate vector in 


$$
\begin{aligned}
& \mathcal{R}_{L}\left(P_{\mathbf{X}} Q_{\mathbf{X}}\right), \\
& I\left(T^{n}, \widetilde{T}^{n} ; U\right)=I\left(X^{n}, Y^{n} ; U\right)+I\left(\widetilde{T}^{n} ; U, T^{n}\right) . \\
& H\left(X^{n}, \widetilde{X}^{n} \mid U\right) \geq H\left(X^{n} \mid U\right)+H\left(\widetilde{X}^{n} \mid U, T^{n}\right) \\
& I\left(Z^{n}, \widetilde{Z}^{n} ; V\right)=I\left(Z^{n} ; V\right)+I\left(\widetilde{Z}^{n} ; V, Z^{n}\right) \\
& H\left(Y^{n}, \widetilde{Y}^{n} \mid U, V\right) \geq H\left(Y^{n} \mid U, V\right)+H\left(\widetilde{Y}^{n} \mid T^{n}, U, Z^{n}, V\right) .
\end{aligned}
$$

Let

$$
\begin{gathered}
\mathbf{R}=\frac{1}{n}\left(I\left(Z^{n} ; V\right), I\left(T^{n} ; U\right)+H\left(X^{n} \mid U\right)+H\left(Y^{n} \mid U, V\right),\right. \\
\left.I\left(T^{n} ; U\right)+H\left(Y^{n} \mid U, V\right)\right) .
\end{gathered}
$$

Define $U^{\prime}=\left(U, T^{n}\right)$ and $V^{\prime}=\left(V, Z^{n}\right)$. Let

$$
\begin{aligned}
\widetilde{\mathbf{R}}=\frac{1}{n} & \left(I\left(\widetilde{Z}^{n} ; V^{\prime}\right), I\left(\widetilde{T}^{n} ; U^{\prime}\right)+H\left(\widetilde{X}^{n} \mid U^{\prime}\right)+H\left(\widetilde{Y}^{n} \mid U^{\prime}, V^{\prime}\right)\right. \\
& \left.+I\left(\widetilde{T}^{n} ; U^{\prime}\right)+H\left(\widetilde{Y}^{n} \mid U^{\prime}, V^{\prime}\right)\right) .
\end{aligned}
$$

Then $\mathbf{R} \in \mathcal{R}_{L}\left(P_{\mathbf{X}}\right)$ and $\widetilde{\mathbf{R}} \in \mathcal{R}_{L}(Q \mathbf{X})$. It then follows that $\mathcal{R}_{L}\left(P_{\mathbf{X}} Q_{\mathbf{X}}\right) \subseteq \mathcal{R}_{L}\left(P_{\mathbf{X}}\right)+\mathcal{R}_{L}\left(Q_{\mathbf{X}}\right)$ and hence we have $\mathcal{R}_{L}\left(P_{\mathbf{X}} Q_{\mathbf{X}}\right)=\mathcal{R}_{L}\left(P_{\mathbf{X}}\right)+\mathcal{R}_{L}\left(Q_{\mathbf{X}}\right)$.

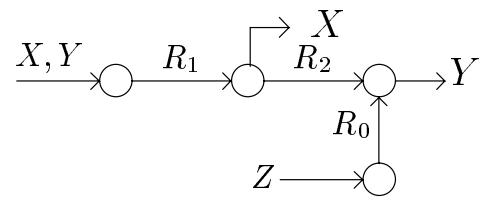

Fig. 3. Multi-Hop Network with coded side information.

\section{General Case}

We begin by showing that the lossless rate region and the rate distortion region for distortion vector $\mathbf{0}$ are the same for finite-alphabet sources and the Hamming distortion measure. This is not a direct consequence of our definitions since lossless source coding requires block error rates to go to zero while $\mathbf{0}$-distortion coding only requires symbol error rates to go to zero. We achieve this by bounding the conditional entropy of one random vector given another in terms of the Hamming distance between them. We use $d_{H}$ to denote the Hamming distance (or Hamming distortion) between any two sequences of the same length, defined as $d_{H}\left(x^{n}, y^{n}\right)=\left|\left\{i \mid x_{i} \neq y_{i}\right\}\right|$.

Lemma 2: Let $V^{n}=\left(V_{1}, V_{2}, \ldots, V_{n}\right)$ be a random vector in $\{0,1, \ldots, m-1\}^{n}$ and let $w$ be the per symbol expected Hamming weight of $V^{n}$, defined as $w:=\frac{1}{n} E d_{H}\left(V^{n}, \mathbf{0}\right)=$ $\frac{1}{n} E\left|\left\{i \mid V_{i} \neq 0\right\}\right|$. Then $H\left(V^{n}\right) \leq n(H(w)+w \log (m-1))$.

Proof. The proof follows from Fano's inequality.

Corollary 1: Let $V^{n}$ and $W^{n}$ be two random vectors in $\{0,1, \ldots, m-1\}^{n}$. Then $\frac{1}{n} H\left(V^{n} \mid W^{n}\right) \leq H(e)+e \log (m-$ $1)=O(e \log (e))$, where $e:=E\left\{\frac{1}{n} d_{H}\left(V^{n}, W^{n}\right)\right\}$.

Proof. Apply Lemma 2 to the random vector $V^{n}-W^{n}$.

Now fix a network source coding problem $\mathcal{N}$. Let $\mathcal{R}_{H}(\mathbf{D})$ denote its rate distortion region using the Hamming distortion measure, and let $\mathcal{R}_{L}$ denote its lossless rate region.
Theorem 2: If the sources are drawn i.i.d., and all source alphabets are finite, then $\mathcal{R}_{H}(\mathbf{0})=R_{L}$.

Proof. Let $m=\max _{1 \leq i \leq s}\left|\mathcal{X}_{i}\right|$. By definition, $\mathcal{R}_{H}(\mathbf{0})\left(\mathcal{R}_{L}\right.$, respectively) consists of all rate vectors for which the bit error rates (block error rates, respectively) can be made arbitrarily small. Therefore, we have immediately that $\mathcal{R}_{L} \subseteq \mathcal{R}_{H}(\mathbf{0})$.

Conversely, let $\mathbf{R} \in \mathcal{R}_{H}(\mathbf{0})$. We next show that for any $\epsilon>0$, the rate vector $\mathbf{R}+\epsilon \mathbf{1}$ is in $\mathcal{R}_{L}$. Choose a length- $n$ block code $\mathcal{C}$ with rate $\mathbf{R}+\frac{\epsilon}{2} \mathbf{1}$ such that all the reproductions have bit error rates no greater than $e(\epsilon)$, where $e(\epsilon)$ is chosen so that

$$
k(H(e(\epsilon))+e(\epsilon) \log (m-1))<\frac{\epsilon}{2} .
$$

Here we recall that $k$ is the total number of reproduction requests, i.e., $k$ is the number of pairs $(v, j)$ where $v \in \mathcal{V}$ and $j \in J(v)$. For each $v \in \mathcal{V}$ with $J(v) \neq \emptyset$ and every $j \in J(v)$, let $\widehat{X}_{j}^{n}(v)$ be the reproduction of $X_{j}^{n}$ at vertex $v$ using code $\mathcal{C}$. For every such $(v, j)$, we choose one vertex $v_{v, j}^{\prime} \in \mathcal{V}$ such that $X_{j}$ can be observed by $v_{v, j}^{\prime}$ and there is a path from $v_{v, j}^{\prime}$ to $v$. We use $\mathcal{P}_{v, j}$ to denote this path. By assumption and Corollary 1 ,

$$
\frac{1}{n} H\left(X_{j}^{n} \mid \widehat{X}_{j}^{n}(v)\right) \leq H(e(\epsilon))+e(\epsilon) \log (m-1) .
$$

Thus the additional rate required to describe $X_{j}^{n}$ along path $\mathcal{P}_{v, j}$ to achieve arbitrarily low block error rate is bounded by $H(e(\epsilon))+e(\epsilon) \log (m-1)$. We therefore achieve lossless coding by applying this approach on all triples $\left(v, j, v_{v, j}^{\prime}\right)$. Since there are $k$ reproduction requests, the resulting rate vector $\mathbf{R}^{\prime}$ satisfies

$$
\mathbf{R}^{\prime} \leq \mathbf{R}+\frac{\epsilon}{2}+k(H(e(\epsilon))+e(\epsilon) \log (m-1)) \mathbf{1} \leq \mathbf{R}+\epsilon \mathbf{1} .
$$

Hence $\mathbf{R}+\epsilon \mathbf{1} \in \mathcal{R}_{L}$.

The following lemma follows directly from Theorem 2 .

Lemma 3:

$$
\mathcal{R}_{L}\left(\lambda P_{\mathbf{X}}+\bar{\lambda} Q_{\mathbf{X}}\right) \subseteq \frac{1}{2} \mathcal{R}_{L}\left(\lambda P_{\mathbf{X}}^{2}+\bar{\lambda} Q_{\mathbf{X}}^{2}\right) .
$$

Since $\mathcal{R}_{L}\left(P_{\mathbf{X}}\right)$ is a subset of $\left\{\mathbf{R} \mid R_{e} \geq 0\right\}$ for any $P_{\mathbf{X}}$, $\lim _{n \rightarrow \infty} \frac{1}{2^{n}} \mathcal{R}_{L}\left(\lambda P_{\mathbf{X}}^{2^{n}}+\bar{\lambda} Q_{\mathbf{X}}^{2^{n}}\right)$ exists. By Lemma 3, we have the following theorem.

Theorem 3:

$$
\mathcal{R}_{L}\left(\lambda P_{\mathbf{X}}+\bar{\lambda} Q_{\mathbf{X}}\right) \subseteq \lim _{n \rightarrow \infty} \frac{1}{2^{n}} \mathcal{R}_{L}\left(\lambda P_{\mathbf{X}}^{2^{n}}+\bar{\lambda} Q_{\mathbf{X}}^{2^{n}}\right)
$$

We conjecture that the limit in Theorem 3 is a subset of $\lambda \mathcal{R}_{L}\left(P_{\mathbf{X}}\right)+\bar{\lambda} \mathcal{R}_{L}\left(Q_{\mathbf{X}}\right)$ for more networks. If true, the conjecture would imply $\mathcal{R}_{L}$ is concave.

\section{REFERENCES}

[1] R. F. Ahlswede and J. Korner, "Source Coding with Side Information and a Converse for Degraded Broadcast Channels," IEEE Trans. Inform. Theory, vol. IT-21, no. 6, pp. 629-637, Nov. 1975.

[2] S. R. Li, R. W. Yeung, and N. Cai, "Linear Network Coding," IEEE Trans. Inform. Theory, vol. IT-49, no. 2, pp. 371-381, Feb. 2003.

[3] T. M. Cover and J. A. Thomas, Elements of Information Theory, WileyInterscience Publication, 1991.

[4] R. Ahlswede, N. Cai and S.-Y. R. Li and R. W. Yeung, "Network Information Flow,' IEEE Trans. Inform. Theory, vol. 46, no. 4, pp. 1204$1216,2000$. 